\title{
INFORMATION RECYCLING MATHEMATICAL METHODS FOR PROTEAN SYSTEMS: A PATH-WAY APPROACH TO A GEOMETRIC PROGRAM
}

\author{
S. Kumar ${ }^{1}$ \\ Department of Mathematics and Statistics, \\ University of Melbourne, Australia \\ skumar@ms.unimelb.edu.au
}

\begin{abstract}
It is assumed that input values in a mathematical model may change frequently due to interactions that may be external or internal and/or may be due to design considerations. This changing environment is typical in a design situation where various possibilities are tried out before accepting one as the final design. Within the changing environment, a solution procedure has been described that attempts to obtain the solution to the changed problem by using the information and results already available from the solution of the problem that was obtained before changes occurred. The author in [15] has called this a 'path-way' approach, and terms all such methods based on this philosophy as "information recycling mathematical methods". In this paper, a geometric program (GP) is considered under a protean environment.
\end{abstract}

\section{OPSOMMING}

Die insetwaardes van ' $n$ wiskundige model verander dikwels as gevolg van interne of eksterne interaksies en/of ontwerpaanpassings. Al te dikwels vind dit plaas wanneer die ontwerpomgewing vereis dat verskeie ontwerpe oorweeg moet word voordat 'n finale besluit gemaak word. Vir hierdie situasie word ' $n$ oplossing beskryf wat tred hou met die milieu van verandering. Die outeur beskryf die metode via die analoog van 'n "voetpad"-benadering, en doop die filosife met die elegante naam "wiskunde sikliese inligtingsmetodes". Geometriese programmering word ingespan in 'n proteusagtige omgewing.

\footnotetext{
${ }^{1}$ Also School of Computer Science and Mathematics, Victoria University, Footscray Park Campus, PO Box 14428, Melbourne City, MC 8001, Australia.
} 


\section{INTRODUCTION}

The concept of developing information recycling methods was described and developed by Kumar [14,15]. In those papers, he considered the case of a linear program, a quadratic program, and a second order DE, and developed algorithmic details for each case. Talukder and Kumar [16], and Talukder, Kumar and Jones [17] developed the information recycling approach for network problems arising in connection with circuits free of specified links. Three terms are central to these developments, which are explained in detail in [15]. These terms are: 'recycling', 'protean system' and 'pathway approach'. Since they are essential for understanding the approach discussed in this paper, a brief explanation of each is given below.

The recycling concept in waste management (Grover [7]) deals with assigning waste from one situation as a resource to some other situation. Similar attempts can be made in the mathematical analysis. The old available information after a model has been analysed is 'waste', since that model is no longer a valid representation of the situation it was intended for. This happens due to changes, and consequently various input values also change. The recycling concept from waste management motivates one to utilise the available information as a free resource for analysis of the changed situation, if possible. This philosophy is labelled 'the information recycling approach'.

The concept of an information recycling approach is appropriate in a 'protean environment' $[11,12,13]$, where input to a model changes due to external and internal interactions. However, in the case of geometric programming models, which are suitable for design problems, changes also arise due to design considerations. The repeated use of a mathematical model with changed input values has been called 'a protean environment' by the author, as discussed in $[11,12,13]$. The objective in this paper is to develop an information recycling method for a geometric programming model in a protean environment.

Finally, the term 'pathway approach' has been used. Usually solution methods create a kind of path from a defined starting point in order to reach the desired end point. However, in the present context, the defined starting point is the solution obtained before changes, and the desired end point is the required solution after changes have occurred in the values of the input parameters. Thus a path has to be created that joins these two solutions, where the starting point is the solution before changes and the terminal point is the solution to the required new problem after changes (see Garcia and Zangwill [6]).

This paper deals with a geometric program model that is solved by using the information recycling philosophy. It is known that a geometric programming model is appropriate for resolving the values of the design parameters; and in accepting a final design it is important that the situation is studied under different scenarios in order to consider various possibilities. Thus repeated application of the same model under different input parameters is possible in a given situation. So the study of a geometric program under a protean environment is desirable, and this paper is an 
attempt to do that.

In section 2, a brief discussion of a geometric programming model and conditions of optimality is offered. A protean GP is presented in section 3. The information recycling approach for a geometric program has been developed in section 4 , and algorithmic details have been provided. Section 5 deals with numerical illustrations.

\section{A GEOMETRIC PROGRAM MODEL}

The optimality conditions for a GP model are summarised in this section. Let a GP model be given by:

Minimize $g(X)=\sum_{i} t_{i}$,

where

$$
\begin{aligned}
& t_{i}=C_{i} x_{1}^{a_{i 1}} x_{2}^{a_{i 2}} \ldots x_{k}^{a_{i k}} \\
& C_{i}>0, x_{1}, x_{2}, \ldots, x_{k}>0 \\
& i=1,2, \ldots, n .
\end{aligned}
$$

Thus all $t_{i}$ are $>0$. The arithmetic-geometric inequality is given by:

$$
\min \left\{\sum_{i=1}^{n} t_{i}\right\} \geq \max \left\{\left(\frac{t_{1}}{\partial_{1}}\right)^{\partial_{1}}\left(\frac{t_{2}}{\partial_{2}}\right)^{\partial_{2}} \ldots\left(\frac{t_{n}}{\partial_{n}}\right)^{\partial_{n}}\right\},
$$

where

$$
\begin{aligned}
& \partial_{1}+\partial_{2}+\ldots+\partial_{n}=1 \\
& \partial_{1}, \partial_{2}, \ldots, \partial_{n}>0 .
\end{aligned}
$$

Alternatively relation (2) is equivalent to:

$$
\begin{aligned}
& \left(C_{1} x_{1}^{a_{11}} x_{2}^{a_{12}} \ldots x_{k}^{a_{1 k}}\right)+\ldots+\left(C_{n} x_{1}^{a_{n 1}} x_{2}^{a_{n 2}} \ldots x_{k}^{a_{n k}}\right) \geq \\
& \left.\left(\frac{C_{1}}{\partial_{1}}\right)^{\partial_{1}}\left(\frac{C_{2}}{\partial_{2}}\right)^{\partial_{2}} \ldots\left(\frac{C_{n}}{\partial_{n}}\right)^{\partial_{n}} x_{1}^{D_{1}} x_{2}^{D_{2}} \ldots x_{k}^{D_{k}}\right)
\end{aligned}
$$

where

$$
\left.\begin{array}{l}
D_{j}=\sum_{i=1}^{n} \partial_{i} a_{i j} \\
j=1,2, \ldots k
\end{array}\right)
$$

For the RHS of relation (4) to attain its maximum value, the conditions of optimality are: 
$D_{j}=\sum_{i=1}^{n} \partial_{i} a_{i j}$

Thus relation (4), when conditions (6) are satisfied, becomes

$$
\left.\begin{array}{l}
\left(C_{1} x_{1}^{a_{11}} x_{2}^{a_{12}} \ldots x_{k}^{a_{1 k}}\right)+\ldots+\left(C_{n} x_{1}^{a_{n 1}} x_{2}^{a_{n 2}} \ldots x_{k}^{a_{n k}}\right) \geq \\
\left(\frac{C_{1}}{\partial_{1}}\right)^{\partial_{1}}\left(\frac{C_{2}}{\partial_{2}}\right)^{\partial_{2}} \ldots\left(\frac{C_{n}}{\partial_{n}}\right)^{\partial_{n}}
\end{array}\right)
$$

Note that in the LHS of (7) the unknowns to be determined are $x_{1}, x_{2}, \ldots, x_{k}$ and $C_{1}, C_{2}, \ldots, C_{n}, \alpha_{i j}, \forall i j$ are the known given values; and the unknowns in the RHS function to be determined are $\partial_{1}, \partial_{2}, \ldots, \partial_{n}$. These $n$ unknown values can be determined from the $k$ number of conditions given by relation (6), and one more condition as given by the relation (3). In addition, all these values are restricted to non-negative values. Thus, if the number of unknowns to be determined $n=k+1$, the situation is labelled as a zero order of difficulty, and when $n>k+1$, the problem is said to have $(n-k-1)$ order of difficulty. When $(n<k)$, the problem is said to be over-constrained. A detailed account of a GP model can be seen in [1].

\section{PROTEAN GP MODEL}

Since values of the known parameters $C_{1}, C_{2}, \ldots, C_{n}$ and $a_{i j}, i=1,2, \ldots, n ; j=1,2, \ldots, k$ in two consecutive considerations are unlikely to remain the same, the values of unknowns $\partial_{1}, \partial_{2}, \ldots, \partial_{n}$ in RHS of (7) will change. The problem is to find new values of $\partial_{1}, \partial_{2}, \ldots, \partial_{n}$. and consequently the new values of the variables $x_{1}, x_{2}, \ldots, x_{k}$. In this section, a pathway approach for a protean GP model is discussed. All parametric values are reviewed for each design scenario, and these scenarios do change for various considerations and possibilities. One may be interested in evaluating these different values under different considerations.

\subsection{Optimality conditions for the GP model in two consecutive intervals}

To distinguish conditions for two consecutive designs, the two sets of conditions for the $i^{\text {th }}$ design and the $(i+1)$ th design are represented by using the following notation.

$$
\begin{aligned}
& { }^{i} D_{j}=\sum_{l=1}^{n}{ }^{i} \partial_{l}{ }^{i} a_{i j}=0, \forall j \\
& { }^{i} \partial_{1}+{ }^{i} \partial_{2}+\ldots+{ }^{i} \partial_{n}=1 \\
& { }^{i} \partial_{1},{ }^{i} \partial_{2}, \ldots,{ }^{i} \partial_{n}>0 . \\
& j=1,2, \ldots, k .
\end{aligned}
$$


and

$$
\begin{aligned}
& { }^{i+1} D_{j}=\sum_{l=1}^{n}{ }^{i+1} \partial_{l}{ }^{i+1} a_{i j}=0, \forall j \\
& { }^{i+1} \partial_{1}+{ }^{i+1} \partial_{2}+\ldots+{ }^{i+1} \partial_{n}=1 \\
& { }^{i+1} \partial_{1},{ }^{i+1} \partial_{2}, \ldots,{ }^{i+1} \partial_{n}>0 . \\
& j=1,2, \ldots, k .
\end{aligned}
$$

Here the superscript to the left of a symbol represents the design scenario or the design number. It is assumed that an optimal solution to the model represented by (2) for the $i^{\text {th }}$ design has been obtained by using any appropriate solution procedure [1]. The solution for conditions (9) requires attention only if some or all values of the various elements $n, k, a_{i j}$ have changed for the $(i+1) t h$ design. Thus the problem is to find a solution to conditions (9) from the solution already obtained to conditions (8) - in other words, to find the path that joins the known point represented by the solution of conditions (8) to a new point that represents the solution of conditions (9). Also note that these conditions are independent of $C_{i}$ values. Once a solution to $\partial_{1}, \partial_{2}, \ldots, \partial_{n}$. is known, one has to find the corresponding new solution for the variables $x_{1}, x_{2}, \ldots, x_{k}$ where $C_{i}$ values play their role.

\subsection{A three-stage approach to find the solution to the problem posed in section 3.1}

In the following the words 'design' and 'interval' are used interchangeably. They represent a periodic review and observation if input values have changed, the reason being interaction or a design scenario. It is assumed that the mathematical problem for the $(i+1)$ th interval still is a GP model; changes arise only with respect to the values of some or all its elements - i.e. values of $n, k, a_{i j}, C_{i}$ might change. This is a realistic assumption, as various considerations are likely to interact in different ways. However, it is certain that the changed values can be expressed by simple addition and subtraction with respect to previous values of those parameters. Thus we can rewrite new values of the elements $n, k, a_{i j}, C_{i}$ as follows:

$$
\begin{aligned}
& { }^{i+1} k={ }^{i} k+\overline{{ }^{i} k} ;{ }^{i+1} n={ }^{i} n+\overline{{ }^{i} n} \\
& { }^{i+1} a_{i j}={ }^{i} a_{i j}+\overline{{ }^{i} a_{i j}}, \forall i j \\
& { }^{i+1} C_{i}={ }^{i} C_{i}+\overline{{ }^{i} C_{i}}, \forall i
\end{aligned}
$$

Here $i=1,2, \ldots n ; j=1,2, \ldots, k$ and $\overline{{ }^{i} c_{i}}, \forall i, \overline{{ }^{i} a_{i j}}, \forall i j,{ }^{i} k, \bar{i}{ }^{i} n$ are unrestricted quantities i.e. positive, negative, or zero known values. To simplify presentation of the above discussed ideas, it is assumed that in two consecutive periods, the number of unknown variables to be determined remains unchanged. Thus conditions (9) can be expressed in two parts. In this new presentation, the part one conditions are identical 
to (8), and the remaining residual values form the part two to take modifications into account, if any. Thus part two is expected to be a sparse structure. Thus using relations (10), conditions (9) can be rewritten as:

$$
\begin{aligned}
& { }^{i+1} D_{j}=\sum_{l=1}^{n}{ }^{i+1} \partial_{l}\left({ }^{i} a_{i j}+\overline{{ }^{i} a_{i j}}\right)=0, \forall j \\
& { }^{i+1} \partial_{1}+{ }^{i+1} \partial_{2}+\ldots+{ }^{i+1} \partial_{n}=1 \\
& { }^{i+1} \partial_{1},{ }^{i+1} \partial_{2}, \ldots .,{ }^{i+1} \partial_{n}>0 . \\
& j=1,2, \ldots, k .
\end{aligned}
$$

Thus with respect to the $i^{\text {th }}$ design conditions (8), an additional sparse structure has been added to those conditions, as shown in (11).

Here, for simplicity, it has been assumed that the number of variables and the number of terms remain the same in two consecutive designs - i.e. ${ }^{i} n={ }^{i+1} n$ and ${ }^{i} k={ }^{i+1} k$, then the number of unknown $\partial_{i}$ 's to be determined remain the same as was the case for the $i^{\text {th }}$ consideration. Information from the $i^{\text {th }}$ design can be used on the non-zero columns of (11) to obtain the corresponding equivalent matrix for the $(i+1)^{\text {th }}$ interval model. Since conditions (10) or equivalently (11) are linear, LP interpretation is possible with an objective function comprised of the artificial variables. The approach is similar to one explained by the author in an earlier paper [15]. However, when columns of ${ }^{i} a_{i j}$ and $\overline{{ }^{i} a_{i j}}$ are combined into one column, they may not be able to hold on to its structure with respect to a LP extreme point interpretation. Furthermore, the feasibility and the optimality conditions with respect to $(i+1)^{\text {th }}$ design problem may not remain satisfied. In other words, it is emphasized that the resulting solution with respect to the $(i+1)^{\text {th }}$ design may not even be an extreme point solution, and may not even be feasible. At this stage, three stage calculations to restore these LP characteristics are used as follows:

\section{Stage 1}

Perform pivoting operations to restore the basis - i.e. for each basis variable in the $i^{\text {th }}$ design, a unit column prevails for the new data with respect to the $(i+1)^{\text {th }}$ design.

\section{Stage 2}

After stage 1 calculations, if optimality conditions are not satisfied, use the simplex iterations to establish optimality conditions.

\section{Stage 3}

In the resulting tableau after stage 2 computations, if the solution does not satisfy the feasibility requirement, use the dual simplex method to obtain a feasible solution, holding on to the optimality conditions. 
The end of stage 3 results in a new solution to values of ${ }^{i+1} \partial_{1},{ }^{i+1} \partial_{2}, \ldots,{ }^{i+1} \partial_{n}$. If these values are $>0$, it is the required new solution for the $(i+1)^{\text {th }}$ design consideration. Recall that it was assumed that ${ }^{i} n={ }^{i+1} n$ and ${ }^{i} k={ }^{i+1} k$, which is not an essential requirement for the proposed method; it was just a simplification for presenting the three-stage calculations, in principle. In the case when one or both conditions are not satisfied, one is required to carry out more modifications, as explained subsequently in the algorithmic steps of the proposed method.

It may also be noted that each tableau in the three stage calculations represents a point in an $n$ dimensional space, which when joined to each other form a piece-wise linear path that joins the solution of the problem for the $i^{\text {th }}$ consideration to the required solution for the $(i+1)^{\text {th }}$ consideration.

\subsection{The Algorithm}

The steps of the algorithm are as follows:

\section{Step 0}

Record from the $i^{\text {th }}$ design model values of:

${ }^{i} n ;{ }^{i} k ;{ }^{i} C_{1},{ }^{i} C_{2}, \ldots,{ }^{i} C_{n} ;{ }^{i} a_{i j}, i=1,2, \ldots,{ }^{i} n ; j=1,2, \ldots,{ }^{i} k$

and the values of ${ }^{i} \partial_{1},{ }^{i} \partial_{2}, \ldots,{ }^{i} \partial_{n}>0$.

0.2 Record the problem data for the $(i+1)^{\text {th }}$ design consideration i.e. values of:

${ }^{i+1} n ;{ }^{i+1} k ;{ }^{i+1} C_{1},{ }^{i+1} C_{2}, \ldots,{ }^{i+1} C_{n} ;{ }^{i+1} a_{i j}, i=1,2, \ldots,{ }^{i+1} n ; j=1,2, \ldots,{ }^{i+1} k$

\section{Step 1}

Check whether the number of variables for the $i^{\text {th }}$ consideration is identical to the number of variables for the $(i+1)^{\text {th }}$ consideration; if so, go to step 2. Otherwise carry out the following modifications:

If a variable is no longer relevant in a design, it can be easily accounted for by using relation (12); but a GP model deals with a design problem, where all variables are strictly required to be $>0$. The situation for (12) may arise only when the design has to be changed with respect to a particular dimension. In that case, we write:

$$
\overline{{ }^{i} a_{i j}}=-{ }^{i} a_{i j}
$$

A more likely change is that a new variable $\overline{{ }^{i+1} \partial_{i}}$ is required for the $(i+1)^{\text {th }}$ consideration compared to $i^{\text {th }}$ consideration, when a new term is added to the LHS of problem (2). In this situation, the new term is simply added to the sparse problem and substituted: 
$\overline{{ }^{i+1} C_{i}}={ }^{i+1} C_{i}$
$\overline{{ }^{i+1} a_{i, n+1}}={ }^{i+1} a_{i, n+1}$

\section{Step 2}

Compare conditions for unknown $\partial_{i}$ for the $i^{\text {th }}$ and $(i+1)^{\text {th }}$ consideration. If the number of terms and number of variables are equal for two consequent considerations, then go to step 3. Otherwise add a new $(n+1)^{\text {th }}$ row and column to an existing $n X n$ matrix. The row is given by:

$$
\begin{aligned}
& { }^{i+1} D_{n+1}=\sum_{l=1}^{n+1}{ }^{i+1} \partial_{l}{ }^{i+1} a_{n+1, l}=0, \\
& { }^{i+1} \partial_{1}+{ }^{i+1} \partial_{2}+\ldots+{ }^{i+1} \partial_{n}+{ }^{i+1} \partial_{n+1}=1 \\
& { }^{i+1} \partial_{1},{ }^{i+1} \partial_{2}, \ldots,{ }^{i+1} \partial_{n},{ }^{i+1} \partial_{n+1}>0 . \\
& j=1,2, \ldots, k .
\end{aligned}
$$

\section{Step 3}

Rearrange the problem for the $(i+1)^{\text {th }}$ design in two parts, as shown in relation (10). If the sparse part of the problem does not exist, the solution for the $i^{\text {th }}$ consideration remains valid for the $(i+1)^{\text {th }}$ consideration. Go to step 6; otherwise go to step 4 .

\section{Step 4}

With respect to the basis of the model for the $i^{\text {th }}$ consideration, perform pivoting calculations on each column of the sparse problem and combine the corresponding column $\mathrm{j}$ with $\bar{j}$. Carry out pivot operations so that the basis with respect to the $i^{\text {th }}$ design is restored. If the resulting solution does not satisfy the feasibility condition, use dual simplex iterations to restore the feasibility of the new solution. When the feasible solution has been obtained, go to step 5 .

\section{Step 5}

Since a basic feasible solution has been obtained, carry out simplex iterations to obtain an optimal solution with respect to the artificial variable objective. Go to step 6.

\section{Step 6}

The current solution is feasible for the $(i+1)^{\text {th }}$ consideration. Print the solution and assign $i+1=i$ and go to Step 0 for the next iteration. 


\section{PROTEAN GP MODELS: ILLUSTRATIVE EXAMPLES}

\section{Example 1}

The purpose of this example is to illustrate the algorithm on a protean GP model in section 3, where input data changes for any reason.

Consider the following GP model for the $i^{\text {th }}$ interval.

Minimize $x_{0}=c_{1} x_{1} x_{2} x_{3}+c_{2} x_{1}^{-1} x_{2}+c_{3} x_{1} x_{3}^{-2}+c_{4} x_{2}^{-3} x_{3}$,

where

$c_{j}>0 \forall j=1,2,3,4$.

$x_{i}>0 \forall i=1,2,3$

Let the above be represented as:

$$
\begin{aligned}
& t_{1}=c_{1} x_{1} x_{2} x_{3}, \\
& t_{2}=c_{2} x_{1}^{-1} x_{2}, \\
& t_{3}=c_{3} x_{1} x_{3}^{-2}, \\
& t_{4}=c_{4} x_{2}^{-3} x_{3}
\end{aligned}
$$

Then the GP inequality gives:

Minimum $\left(t_{1}+t_{2}+t_{3}+t_{4}\right) \geq \operatorname{maximum}\left[\left(\frac{c_{1}}{\partial_{1}}\right)^{\partial_{1}}\left(\frac{c_{2}}{\partial_{2}}\right)^{\partial_{2}}\left(\frac{c_{3}}{\partial_{3}}\right)^{\partial_{3}}\left(\frac{c_{4}}{\partial_{4}}\right)^{\partial_{4}}\right]$

The conditions for optimality are:

$\partial_{1}+\partial_{2}+\partial_{3}+\partial_{4}=1$

$\partial_{1}-\partial_{2}+\partial_{3}=0$

$\partial_{1}+\partial_{2}-3 \partial_{4}=0$

$\partial_{1}-2 \partial_{3}+\partial_{4}=0$

This zero degree difficulty problem has a solution given by $x_{1}=0.5, x_{2}=1.0, x_{3}=0.5$, which has been obtained from (18) given by $\partial_{1}=1 / 5, \partial_{2}=2 / 5, \partial_{3}=1 / 5, \partial_{4}=1 / 5$. Assume that this solution of relations (18) was obtained by using the LP approach. Variables $\partial_{1}, \partial_{2}, \partial_{3}, \partial_{4}$ are represented by $\mathrm{X} 1, \mathrm{X} 2, \mathrm{X} 3$ and X4 for the phase one calculations when four artificial variables A1, A2, A3, A4 were added to relations (18). The first and last table of the simplex iteration is given below: 


\begin{tabular}{|c|c|c|c|c|c|c|c|c|c|}
\hline Row & X1 & X2 & X3 & X4 & A1 & A2 & A3 & A4 & RHS \\
\hline Obj & 0 & 0 & 0 & 0 & -1 & -1 & -1 & -1 & -1 \\
\hline A1 & 1 & 1 & 1 & 1 & 1 & 0 & 0 & 0 & 1 \\
\hline A2 & 1 & -1 & 1 & 0 & 0 & 1 & 0 & 0 & 0 \\
\hline A3 & 1 & 1 & 0 & -3 & 0 & 0 & 1 & 0 & 0 \\
\hline Row & 1 & 0 & -2 & 1 & 0 & 0 & 0 & 1 & 0 \\
\hline
\end{tabular}

Table 1: Initial information with respect to (18)

\begin{tabular}{|c|c|c|c|c|c|c|c|c|c|}
\hline Row & X1 & X2 & X3 & X4 & A1 & A2 & A3 & A4 & RHS \\
\hline X1 & 1 & 0 & 0 & 0 & $1 / 5$ & $9 / 25$ & $4 / 25$ & $7 / 25$ & $1 / 5$ \\
\hline X2 & 0 & 1 & 0 & 0 & $2 / 5$ & - & $3 / 25$ & $-1 / 25$ & $2 / 5$ \\
\hline & & & & & & $12 / 25$ & & & \\
\hline X3 & 0 & 0 & 1 & 0 & $1 / 5$ & $4 / 25$ & $-1 / 25$ & $-8 / 25$ & $1 / 5$ \\
\hline X4 & 0 & 0 & 0 & 1 & $1 / 5$ & $-1 / 25$ & $-6 / 25$ & $2 / 25$ & $1 / 5$ \\
\hline
\end{tabular}

Table 2: Final table giving values of required variables and inverse of the basic matrix

Now consider that the problem in the $(i+1)^{\text {th }}$ interval become

$$
\begin{aligned}
& t_{1}=c_{1} x_{1} x_{2} x_{3}, \\
& t_{2}=c_{2} x_{1}^{-1} x_{2} x_{3}, \\
& t_{3}=c_{3} x_{1} x_{3}^{-3}, \\
& t_{4}=c_{4} x_{1}^{-2} x_{2}^{-3} x_{3}
\end{aligned}
$$

Thus new conditions for values of ${ }^{i+1} \partial_{i}>0$, if the solution exists, are:

$$
\begin{aligned}
& { }^{i+1} \partial_{1}+{ }^{i+1} \partial_{2}+{ }^{i+1} \partial_{3}+{ }^{i+1} \partial_{4}=1 \\
& { }^{i+1} \partial_{1}-{ }^{i+1} \partial_{2}+{ }^{i+1} \partial_{3}-2^{i+1} \partial_{4}=0 \\
& { }^{i+1} \partial_{1}+{ }^{i+1} \partial_{2}-3^{i+1} \partial_{4}=0 \\
& 2^{i+1} \partial_{1}+{ }^{i+1} \partial_{2}-3^{i+1} \partial_{3}+{ }^{i+1} \partial_{4}=0
\end{aligned}
$$

Rewriting (20) in the two parts by using the relation (4), we get

Maximize $x_{0}=-(A 1+A 2+A 3+A 4)$

Subject to

$$
\begin{aligned}
& { }^{i} \partial_{1}+{ }^{i} \partial_{2}+{ }^{i} \partial_{3}+{ }^{i} \partial_{4}+0\left(\bar{i}^{\bar{i}}+\overline{{ }^{i} \partial_{2}}+\overline{{ }^{i} \partial_{3}}+\overline{{ }^{i} \partial_{4}}\right)=1 \\
& { }^{i} \partial_{1}-{ }^{i} \partial_{2}+{ }^{i} \partial_{3}+0^{i} \partial_{4}+0 \overline{{ }^{1} \partial_{1}}+0^{\bar{i} \partial_{2}}+0^{\bar{i} \partial_{3}}-2^{i} \partial_{4}=0 \\
& { }^{i} \partial_{1}+{ }^{i} \partial_{2}+0^{i} \partial_{3}-3^{i} \partial_{4}+0^{i} \partial_{1}+0^{i} \partial_{2}+0^{i} \partial_{3}+0^{i} \partial_{4}=0
\end{aligned}
$$


${ }^{i} \partial_{1}+0^{i} \partial_{2}-2^{i} \partial_{3}+{ }^{i} \partial_{4}+\overline{{ }^{i} \partial_{1}}+\overline{{ }^{i} \partial_{2}}-\overline{{ }^{i} \partial_{3}}+0 \overline{{ }^{i} \partial_{4}}=0$

Corresponding to variables ${ }^{i} \partial_{1},{ }^{i} \partial_{2},{ }^{i} \partial_{3},{ }^{i} \partial_{4}$ in relations (21) the inverse matrix from

Table 2 is known. We use this inverse matrix to find the corresponding columns for new variables $\overline{{ }^{i} \partial_{1}}, \bar{i} \partial_{2}, \overline{{ }^{i} \partial_{3}}, \overline{{ }^{i} \partial_{4}}$ by using the relation:

$B^{-1} A_{j}$

The value of $B^{-1}$ is given below:

\begin{tabular}{|l|l|l|l|}
\hline A1 & A2 & A3 & A4 \\
\hline $1 / 5$ & $9 / 25$ & $4 / 25$ & $7 / 25$ \\
\hline $2 / 5$ & - & $3 / 25$ & $-1 / 25$ \\
\hline & $12 / 25$ & & \\
\hline $1 / 5$ & $4 / 25$ & $-1 / 25$ & $-8 / 25$ \\
\hline $1 / 5$ & $-1 / 25$ & $-6 / 25$ & $2 / 25$ \\
\hline
\end{tabular}

Table 3: The inverse matrix elements

From (21) column elements with respect to four new variables, $\bar{i} \partial_{1}, \bar{i}{ }^{i} \partial_{2}, \bar{i}{ }^{i} \partial_{3}, \bar{i} \partial_{4}$, are respectively given by:

$\left[\begin{array}{c}\bar{i} \partial_{1} \\ 0 \\ 0 \\ 0 \\ 1\end{array}\right],\left[\begin{array}{c}\bar{i} \partial_{2} \\ 0 \\ 0 \\ 0 \\ 1\end{array}\right],\left[\begin{array}{c}\bar{i} \partial_{3} \\ 0 \\ 0 \\ 0 \\ -1\end{array}\right]$ and $\left[\begin{array}{c}\bar{i} \partial_{4} \\ 0 \\ -2 \\ 0 \\ 0\end{array}\right]$

Using relation (22), Table 3 and column vectors (23), we get the corresponding column vectors as given below in (24):

$\left[\begin{array}{c}7 / 25 \\ -1 / 25 \\ -8 / 25 \\ 2 / 25\end{array}\right],\left[\begin{array}{c}7 / 25 \\ -1 / 25 \\ -8 / 25 \\ 2 / 25\end{array}\right],\left[\begin{array}{c}-7 / 25 \\ 1 / 25 \\ 8 / 25 \\ -2 / 25\end{array}\right]$, and $\left[\begin{array}{c}-18 / 25 \\ 24 / 25 \\ -8 / 25 \\ 2 / 25\end{array}\right]$.

Thus new values will be as shown in Table 4 .

$\begin{array}{llllllllllllll}\text { Row } & \text { X1 } & \text { X2 } & \text { X3 } & \text { X4 } & \underline{X} 1 & \underline{X} 2 & \underline{X} 3 & \underline{X} & \text { A1 } & \text { A2 } & \text { A3 } & \text { A4 } & \text { RHS } \\ \text { X1 } & 1 & 0 & 0 & 0 & 7 / 25 & 7 / 25 & -7 / 25 & -18 / 25 & 1 / 5 & 9 / 25 & 4 / 25 & 7 / 25 & 1 / 5 \\ \text { X2 } & 0 & 1 & 0 & 0 & -1 / 25 & -1 / 25 & 1 / 25 & 24 / 25 & 2 / 5 & -12 / 25 & 3 / 25 & -1 / 25 & 2 / 5 \\ \text { X3 } & 0 & 0 & 1 & 0 & -8 / 25 & -8 / 25 & 8 / 25 & -8 / 25 & 1 / 5 & 4 / 25 & -1 / 25 & -8 / 25 & 1 / 5 \\ \text { X4 } & 0 & 0 & 0 & 1 & 2 / 25 & 2 / 25 & -2 / 25 & 2 / 25 & 1 / 5 & -1 / 25 & -6 / 25 & 2 / 25 & 1 / 5\end{array}$

Table 4: Values with respect to the $(i+1)^{\text {th }}$ interval problem. 
Combining columns of $\mathrm{Xl}$ and $\overline{X l}$ for $\mathrm{l}=1,2,3$, and 4 we obtain Table 5, given below:

$\begin{array}{llllllllllll}\begin{array}{l}\text { New } \\ \text { Value }\end{array} & \mathrm{X} 1+\underline{\mathrm{X} 1} & \mathrm{X} 2+\underline{\mathrm{X} 2} & \mathrm{X} 3+\underline{\mathrm{X} 3} & \mathrm{X} 4+\underline{\mathrm{X} 4} & \mathrm{~A} 1 & \mathrm{~A} 2 & \mathrm{~A} 3 & \mathrm{~A} 4 & \mathrm{RHS} \\ \mathrm{X} 1 & 32 / 25 & 7 / 35 & -7 / 25 & -18 / 25 & 1 / 5 & 9 / 25 & 4 / 25 & 7 / 25 & 1 / 5 \\ \mathrm{X} 2 & -1 / 25 & 24 / 25 & 1 / 25 & 24 / 25 & 2 / 5 & -12 / 25 & 3 / 25 & -1 / 25 & 2 / 5 \\ \mathrm{X} 3 & -8 / 25 & -8 / 25 & 33 / 25 & -8 / 25 & 1 / 5 & 4 / 25 & -1 / 25 & -8 / 25 & 1 / 5 \\ \mathrm{X} 4 & 2 / 25 & 2 / 25 & -2 / 25 & 27 / 25 & 1 / 5 & -1 / 25 & -6 / 25 & 2 / 25 & 1 / 5\end{array}$

Table 5: Where $\mathrm{X} 1+\overline{X l}, \mathrm{l}=1,2,3,4$ represent new variable Xl for the $(i+1)^{\text {th }}$ interval

Since we have lost all unit column structure with respect to the variables X1, X2, X3, and $\mathrm{X} 4$, first these four columns are restored to the unit column structure by using the elementary operations on elements of Table 5, and with rearrangement it is as given in Table 6.

$\begin{array}{lllllllllll}\begin{array}{l}\text { New } \\ \text { Value }\end{array} & \mathrm{X} 1+\underline{\mathrm{X} 1} & \mathrm{X} 2+\underline{\mathrm{X} 2} & \mathrm{X} 3+\underline{\mathrm{X} 3} & \mathrm{X} 4+\underline{\mathrm{X} 4} & \mathrm{~A} 1 & \mathrm{~A} 2 & \mathrm{~A} 3 & \mathrm{~A} 4 & \mathrm{RHS} \\ \mathrm{X} 1 & 1 & 0 & 0 & 0 & 11 / 41 & 16 / 41 & -4 / 41 & 9 / 41 & 11 / 41 \\ \mathrm{X} 2 & 0 & 1 & 0 & 0 & 10 / 41 & -19 / 41 & 15 / 41 & -3 / 41 & 10 / 41 \\ \mathrm{X} 3 & 0 & 0 & 1 & 0 & 13 / 41 & 4 / 41 & -1 / 41 & -8 / 41 & 13 / 41 \\ \mathrm{X} 4 & 0 & 0 & 0 & 1 & 7 / 41 & -1 / 41 & -10 / 41 & 2 / 41 & 7 / 41\end{array}$

Table 6: Restored equivalent values from Table 5.

Since all feasibility and optimality conditions are satisfied, the resulting new solution for the $(i+1)^{\text {th }}$ consideration, is given by $X 1=\partial_{1}=11 / 41, X 2=\partial_{2}=10 / 41, X 3=\partial_{3}=13 / 41, X 4=\partial_{4}=7 / 41$.

Once these new $\partial_{1}, \partial_{2}, \partial_{3}, \partial_{4}$ values are known, one is required to find the value of the RHS of (17) for the $(i+1)^{\text {th }}$ consideration. In the above example, 16.697 is the new maximum value of the RHS function. Once this is known, one has to return to the original problem and apply to each term the relation:

$t_{i}=16.697 \partial_{i} ; i=1,2,3,4$.

Using relations (25), the values of the variables $x_{1}, x_{2}, x_{3}$ are determined for the model given by relation (19). For the above example, these new values are given by $x_{1}=0.19927, x_{2}=0.48048, x_{3}=0.42224$ to five significant decimal places. 


\section{Example 2}

The purpose of this example is to illustrate various design considerations before accepting a particular design. Consider that a container is to be designed to hold 300 cubic $\mathrm{cm}$ of liquid. This example is a slight modification of an example discussed in [1], where only the cylindrical design was considered. Since a large number of units of this product would be required, the minimum cost per unit is extremely important for the organization. Let the material cost for the walls be $\$ 0.20$ per sq $\mathrm{cm}$, whereas the cost of material used for the bottom and top of this container is $\$ 0.80$ per sq cm. The cost of manufacturing containers is independent of the shape of the container.

\section{Case 1}

Consider a circular cylindrical tube of radius ' $r$ ' and height ' $h$ '. The cost function to be minimised, as given in [1], is given by:

$$
T C_{c y l i n d e r}=120 r^{(-1)}+0.8 \pi\left(r^{2}\right)=C_{1} r^{(-1)}+C_{2} r^{(2)}
$$

where $C_{1}=120$ and $C_{2}=0.8 \pi$. The optimality conditions are given by

$$
\begin{aligned}
& -\delta_{1}+2 \delta_{2}=0 \\
& \delta_{1}+\delta_{2}=1
\end{aligned}
$$

The solution for the above equations is $\delta_{1}=2 / 3, \delta_{2}=1 / 3$, i.e. the optimal cost is distributed such that $2 / 3$ of the total cost is towards the walls and $1 / 3$ is for the top and bottom. This results in total cost $=\left[\left(\frac{120}{2 / 3}\right)^{(2 / 3)} \cdot\left(\frac{0.8 \pi}{1 / 3}\right)^{(1 / 3)}\right]=62.513$.

\section{Case 2}

Consider a container with a triangle, each side of length equal to 'a' and height ' $h$ '.

$$
T C_{\Delta b a s e}=\left(\frac{720}{\sqrt{3}}\right) a^{-1}+(0.2)(\sqrt{3}) a^{2}=C_{1 \Delta} a^{-1}+C_{2 \Delta} a^{2}
$$

Note that expressions (26) and (28) are similar, hence optimality conditions do not change and the solution remains the same - i.e. $\delta_{1}=2 / 3, \delta_{2}=1 / 3$. Thus for the triangular base design, the cost would be: $\left(\frac{\frac{720}{2 / 3}}{2 / 3}\right)^{(2 / 3)} \cdot\left(\frac{0.4 \sqrt{3}}{1 / 3}\right)^{(1 / 3)}=73.928$

\section{Case 3}

Container with square base of side 'a' and height ' $h$ '. The cost function is given by: 
$T C_{\text {squarebase }}=240 a^{-1}+1.6 a^{2}=C_{1 s q u r e} a^{-1}+C_{2 \text { squre }} a^{2}$.

Once again the cost equations for different designs are the same, so the solution remain unchanged - i.e. $\delta_{1}=2 / 3, \delta_{2}=1 / 3$. Thus the optimal cost for this design would be:

$\left(\frac{240}{(2 / 3)}\right)^{(2 / 3)} \cdot\left(\frac{0.8}{(1 / 3)}\right)^{(1 / 3)}=67.755$

Thus among these three designs, a circular base is the least expensive. Other designs can be evaluated in a similar way.

Note that for the same weight, if one has to create a large visual impression, a triangle base would be preferred. Reconsider the above design problem and assume that 100 grams of material is to be packed in one of the shapes discussed above - i.e. circular, triangular, or square base. Assume that density is 1 per $\mathrm{cm}^{3}$. Relevant information has been summarised in the Table below.

\begin{tabular}{|c|c|c|}
\hline $\begin{array}{c}\text { Shape of packet } \\
\text { Circular base tube }\end{array}$ & $\begin{array}{c}\text { Function to be minimised } \\
200 r^{-1}+2 \pi r^{2}\end{array}$ & Surface Area \\
& $\equiv\left(\frac{200}{2 / 3}\right)^{2 / 3} \cdot\left(\frac{2 \pi}{1 / 3}\right)^{1 / 3}$ & 119.265 \\
\hline Triangular base tube & $\left(\frac{1200}{\sqrt{3}}\right) r^{-1}+\left(\frac{\sqrt{3}}{2}\right) r^{2}$ & 141.045 \\
$\equiv\left(\frac{1200 / \sqrt{3}}{2 / 3}\right)^{2 / 3} \cdot\left(\frac{\sqrt{3} / 2}{1 / 3}\right)^{1 / 3}$ & \\
\hline Square Base tube & $\begin{array}{c}400 r^{-1}+2 r^{2} \\
\left(\frac{400}{2 / 3}\right)^{2 / 3} \cdot\left(\frac{2}{1 / 3}\right)^{1 / 3}\end{array}$
\end{tabular}

Table 7: Surface areas for a uniform quantity in three different shapes

Thus the triangular shape has the maximum surface area - and that may be the reason that the various-sized chocolates with the brand name "Toblerone" are manufactured in triangular shapes, for better visual effect.

\section{DISCUSSION AND REMARKS}

1. The suggested approach uses a dichotomy to relate present values to the immediate past values.

2. By using the approach discussed in this paper, it may be concluded that optimal 
values of $\partial_{1}, \partial_{2}, \ldots, \partial_{n}$ to all GPs are connected by a piece-wise linear path.

3. This solution approach may be desirable for a problem in an experimental stage when one is concerned about various values of design parameters before implementing a final design.

4. If changes are relatively fewer, this approach may result in computational savings. See Example 2 discussed in section 4, where optimality conditions for the GP models for the three designs did not change. Thus, for each of the subsequent considerations, the previous solution remained valid. However, no definite general statement can be made at this stage. Further testing and supporting software is necessary before making a definite conclusion.

5. The idea of information recycling methods can be traced in many existing methods described in the literature - for example, the time series analysis $[8,9]$ recurrence equations used in dynamic programming and related control processes $[2,3,4]$. Further, many earlier approaches discussed in [5,8,10] may also be classified as information recycling methods. We believe this idea is likely to generate more interest in further research to make the mathematical methods more meaningful for industrial applications.

6. For further research, we have to look again at as many mathematical methods as possible, and develop appropriate methods to consider their analysis in the protean situation arising in real life applications. In my opinion, the field is open for research, and your imagination is the limit. For non-linear cases, the challenge is much more complex as non-linear functions are much more sensitive to changes, compared with linear functions.

7. Problem-solving is like winning a war. This analogy was established in $[15,18]$. In this paper, the aim is to emphasize that a model is a representation of a business situation, which changes frequently - like a war situation. Therefore, to be able to deal with a realistic situation a model must be able to absorb shocks that arise due to unexpected interactions. How bumpy a business ride can be is clearly reflected in the following two quotes from a recent book, Straight from the gut by Jack Walsh, a brilliant business magician [18]:

1. Page XV prologue: "There is no perfect business story. I believe that business is a lot like a world-class restaurant. When you peek behind the kitchen doors, the food never looks as good as when it comes to your table on fine china perfectly garnished. Business is messy and chaotic."

2. Page 377: "Being a CEO is the nuts! A whole jumble of thoughts come to mind: Over the top. Wild. Fun. Outrageous. Crazy. Passion. Perpetual motion. The give-and-take. ... Big decisions in the real game. Crises and pressure. Lots of swings. A few home runs. The thrill of winning. The pain of losing.” 
Note that models are to help these business executives who find themselves always on a roller-coaster ride. If the model is intended to help them, it has got to be able to ride on the same roller-coaster. This paper attempts analysis of a geometric program in a changing environment.

\section{ACKNOWLEDGEMENTS}

Thanks are due to anonymous referees for their constructive suggestions.

\section{REFERENCES}

[1] C.S. Beightler and D.T. Philips, Applied Geometric Programming, John Wiley \& Sons, 1976.

[2] R. Bellman, Dynamic programming, Princeton University Press, 1957.

[3] R. Bellman and S. Dreyfus, Applied Dynamic Programming, Princeton University Press, 1962.

[4] S.C. Fang and S. Puthenpura, Linear Optimisation and Extensions - Theory and Algorithms, Prentice Hall, 1993.

[5] W.A Fuller, Introduction to Statistical Time Series, John Wiley and Sons, 1996.

[6] C.B. Garcia and W.I. Zangwill, Pathways to Solutions, Fixed Points, and Equilibria, Prentice Hall Series in Computational Mathematics, 1981.

[7] V.I. Grover, V.K. Grover and W. Hogland, Recovering Energy from Waste: Various Aspects, Science Publishers, Enfield (NH), USA, 2002.

[8] J.D. Hamilton, Time Series Analysis, Princeton University Press, 1994.

[9] M.S. Klamkin, Mathematical Modelling, Classroom Notes in Applied Mathematics, SIAM, 1987.

[10] V. Klee and G.L. Minty, How good is the Simplex Method? Inequalities III, Ed. O.Shisha, Academic Press, New York, 159-179, 1972.

[11] S. Kumar, Optimisation of Protean Systems - A Review, APORS 1994, (Eds.) M. Fushimi and K. Tone, World Scientific Publishers, pp.139-146, 1995.

[12] S. Kumar, H. Arora, C. Chiera and G. Raviganesh, Paths for self-healing Protean Network, Padma Bhusan Professor B.N. Prasad Birth Centenary Commemorative volume published by the Allahabad Mathematical Society, Bulletin of Allahabad Mathematical Society, Vol. 14, pp79-94, 1999.

[13] S. Kumar, G. Raviganesh, J. McPhee and T.M. Nyathi, Terminal Pair reliability in a Protean Network, International Journal of Operations and Quantitative management, Vol. 5, No. 2, pp 133-150, 1999.

[14] S. Kumar, Information Recycling Mathematical methods: A Philosophy and Challenge, Chapter 3 in Importance of Mathematical Modelling of Biological and Biomedical Processes, Eds. LS Luboobi, JYT Mugisha and J. Kasozi, African Society of Biomathematics, Series No. 1, Makerere University Press, Kampala, Uganda, pp 31-46, 2004.

[15] S. Kumar, Information Recycling Mathematical methods for Protean Systems: A Path-way Approach, South African Journal of Industrial Engineering, Vol. 16, No. 2, pp 81-101, 2005.

[16] HM Talukder and S. Kumar, Circuits free of specified links in a protean distribution network, Discovery and Innovation, Vol. 17, Nos. 1 and 2, pp37- 
44, 2005.

[17] HM Talukder, S. Kumar and BC Jones, Information Recycling Mathematical Methods: Analysis of a link weighted protean communication flow networks, to appear in Indian Journal of Mathematics, Vol. 48, No. 2, 2006. .

[18] Sun Tzu, The art of war, Shambhala Dragon Editions, 1988.

[19] J. Welsh, Straight from the gut, Warner Business books, 2001. 